\title{
Bioprocessing of Tissues using Cellular Spheroids
}

Timothy R Olsen ${ }^{1}$ and Frank Alexis ${ }^{1,2^{*}}$

${ }^{1}$ Department of Bioengineering, Clemson University, 301 Rhodes Research Center, Clemson, SC 29634, USA

${ }^{2}$ Institute of Biological Interfaces of Engineering, Department of Bioengineering, Clemson University, 401-2 Rhodes Engineering Research Center, Clemson, SC 29634, USA

"Corresponding author: Frank Alexis, Department of Bioengineering, Clemson University, 301 Rhodes Research Center, Clemson, SC 29634, USA, Tel: (864) 656-5003; Fax: (864) 656-4466; E-mail: falexis@clemson.edu

Rec date: Mar 07, 2014, Acc date: Mar 08, 2014, Pub date: Mar 14, 2014

Copyright: (C) 2014 Alexis F, et al. This is an open-access article distributed under the terms of the Creative Commons Attribution License, which permits unrestricted use, distribution, and reproduction in any medium, provided the original author and source are credited.

\section{Introduction}

Tissue engineering combines the principles of engineering and life sciences in order to repair, restore and regenerate tissues and organs that mimic the functionality and mechanical properties of native tissues [1,2]. In the United States, there are 121,000 people currently waiting for lifesaving organ transplants (kidney, liver, heart) and tissue engineering offers new hope and therapeutic opportunities for these patients $[3,4]$. The classic approach to tissue engineering involves seeding living cells onto a solid biocompatible and eventually a biodegradable scaffold, and then culturing the tissue engineered construct in a bioreactor until the tissue achieves the desired cell density and mechanical properties for implantation [2]. However, new approaches that build 3D tissues with a bottom up approach using cellular spheroids are offering new possibilities. Rapid tissue fabrication approaches using spheroids have three key bioprocessing steps that lead to the development of 3D tissue structures: (1) preprocessing, (2) processing, and (3) post-processing.

In this editorial, we discuss tissue engineering in the context of bioprocessing and biotechnique approaches and the challenges for advancing these processing technologies towards the clinical stage.

\section{Step One: Pre-processing}

Pre-processing involves preparing the building block tissues that are used to ultimately fabricate the 3D tissue. Here, cellular spheroids are the building blocks being used as the 'bio-ink.' Spheroids are attractive for tissue fabrication techniques due to having precise control over cell and extracellular matrix (ECM) composition, the ability for upscaled production and repeatability, their three-dimensional nature and the fact that spheroids will produce their own ECM over time. Spheroids with tissue-specific functions have been fabricated for cardiac, vascular, cartilage, bone, hepatic and pancreatic applications, demonstrating their potentially broad impact for tissue fabrication. In addition to tissue engineering applications, spheroids are used in studying cancer biology and also in pharmaceuticals with high throughput toxicology and drug testing.

There are many pre-processing methods used to prepare cellular spheroids, which include the pellet or re-aggregation culture, spinner culture, rotating wall vessels, cell sheet techniques, liquid overlay, microfluidics, external forces, the hanging drop technique, and micromolded hydrogels [5]. The most popular spheroid pre-processing methods are re-aggregation, hanging drop and micro-molds. In the reaggregation method, cells from a confluent flask are collected and centrifuged down to form a cell pellet in a tube [5]. This pellet can be removed from the tube, cut into fragments and then incubated until they form rounded spheroids. In the hanging drop technique, small volumes $(15-30 \mu \mathrm{L})$ of cell suspensions are pipetted onto the lids of petri dishes [5]. The lid is then inverted, with the drops staying attached due to surface tension. Gravity causes the cell suspension to concentrate at the bottom of the droplet, forming a spheroid. Micromold pre-processing utilizes computer software for rapid prototyping of 'templates' for creating micro-molds with an array of cylindrical pegs with rounded tops. These micro-molds are then used to cast nonadhesive hydrogels into which cells can be seeded into. Due to the non-adhesive surface, the cells will aggregate and form a spheroid.

Spheroids can be fabricated with a either single or multiple cell types, which demonstrates the potential for fabricating many types of tissues. Even more, endothelial cells can be co-cultured with other cell types, like smooth muscle cells, to create uniluminal spheroids [6]. This is important for tissue engineering because it highlights the potential for using uniluminal vascular spheroids as modules for fabricating blood vessels. ECM proteins, like collagen or fibronectin, can be incorporated into cellular spheroids to promote cell-matrix interactions and provide an early structural network for the developing spheroids. Magnetic nanoparticles (MNPs) have also been incorporated into cellular spheroids to allow for the ability to pattern and align the spheroids for tissue assembly. This pre-processing development has allowed for tissues to be manipulated with physical magnetic forces at a distance in the processing step and has potential for being used for maturation techniques.

Millions of tissue spheroids will be required for organ fabrication [7]. High throughput methods for spheroid fabrication have been developed for biological research, therapeutic testing and 3D tissue construction [8-10]. Micro-mold arrays are leading this thrust, with hanging drop and round-bottom well plate designs for fabricating up to 384 spheroids per standard 96 well plate. The use of these approaches, partnered with a robotic dispenser increases the productivity of spheroid biofabrication [7]. After formation, spheroids can be stored in bio-cartridges (micropipettes) for dispensing in the processing step, or stored in cell culture medium for a desired incubation period. Preventing unwanted fusion of stored tissue spheroids will be challenge that develops with upscaling spheroid fabrication.

Ultimately, the pre-processing step allows for customization of the spheroid for specific applications because of the control over cell type, size, and ECM content. Given this precise control over composition and their versatility, spheroids are a desirable candidate for the preprocessing step in rapid fabrication of tissues.

\section{Step Two: Processing}

The processing step involves dispensing the cultured spheroids into the desired geometry, one layer at a time. This step is critical because a bioprocessing technique is required to take individual spheroids and 
position them on a suitable substrate for tissue formation. In this processing step, the spheroids can be dispensed into a 'bio-paper,' which is a biocompatible hydrogel sheet that provides a non-adhesive surface for the spheroids to be printed on $[1,11,12]$. The bio-paper has been mimicked after the natural ECM and contains the minimal composition for cell attachment, growth and maintaining tissue liquidity for permitting fusion of the spheroids. Crosslinked agarose and gelatin have been shown to function well as the bio-paper, as both provide the required non-adhesive surface for the tissues to be printed onto [11]. The bio-paper can also be molded into desired geometries with a peg and circular well, which allows tubular structures to be formed. Standard non-treated, non-adhesive polystyrene plates are used for culturing spheroids long term, as well as assembling tissues [13-15]. Ideally, the bio-paper should mimic the stiffness of the tissue being fabricated.

In order to dispense spheroids for patterning and alignment, robotic pipette dispensers, like the EpMotion-5070 (Eppendorf), have been developed [7]. Developments in rapid prototyping technologies has allowed for robotic bioprinters to be able to continuously dispense spheroids using $\mathrm{x}-\mathrm{y}-\mathrm{z}$ precise robotic position, automated spheroid dispensers, and computer based software [7]. While these devices have been shown to successfully pattern the spheroids, these devices have issues when building tissues up in the $\mathrm{z}$ direction without the use of a mold. Bioprinters are usually custom built, based on the need of the laboratory, and have not yet been standardized in industry. Given this, as lot of tissue assembly is 'done by hand', meaning the spheroids are dispensed into the biopaper or molds in a non-automated fashion using a pipette. To 'build' tissues, spheroids can be dispensed on top of each other in a layer by layer fashion on a biopaper using a bioprinter spheroids can be dispensed into molds and plates with predefined geometries, or magnetically labeled spheroids can be patterned and aligned using magnetic fields [7,13-15].

After the spheroids have been patterned and aligned into the desired geometry, passive contacts from cell-cell and cell-matrix interactions will cause the spheroids to fuse together to reduce configurational energy. Fusion occurs when adjacent spheroids make contact and coalesce into a cohesive tissue, and this is an essential process for tissue maturation. Macroscopically, individual spheroids should no longer be visualized in a fused tissue. While bioprinters and micro-mold designs create passive contact between spheroids, they do nothing to accelerate spheroid fusion, which is critical for tissues manipulation during maturation. Recently, magnetic fields have been used as a physical force to accelerate the fusion process with active contacts by increasing cell-cell and cell-matrix interactions in magnetic cellular spheroids [15]. Mattix et. al. demonstrated that magnetic cellular spheroids whose fusion was mediated by magnetic forces produced a more cohesive and homogenous tissue at earlier time points, when compared to control spheroids without magnetic forces. The use of magnetic forces for accelerating the fusion of magnetic cellular spheroids is a critical improvement because these fused tissues can be introduced into post-processing methods for maturation at earlier time points than previously.

\section{Step Three: Post-processing}

The post-processing step involves using bioprocessing techniques for promoting tissue maturation. Tissues fabricated with spheroids are often associated with poor mechanical properties. Increasing the synthesis, deposition, cross-linking and assembly of ECM is critical for accelerating the maturation of fused tissues because it develops the dense structural network that translates to tissue strength $[1,12]$.

To accelerate the maturation of fused tissues, bioreactors have been designed to simulate in vivo conditions and provide the necessary chemical and mechanical cues experienced by native tissues. Some key characteristics of bioreactors include being able to: (1) control the environment during mechanical stimulation; (2) stimulate multiple constructs with identical or individual waveforms; (3) deliver precise displacements, including those that mimic in vivo activities of daily living; and (4) adjust displacement patterns based on reaction loads and biological activity [16]. Bioreactor types include spinner flask bioreactors for cell seeding on 3D scaffolds, rotating wall vessels for providing a dynamic culture environment with low shear and high mass transfer, hollow-fiber bioreactors for enhancing mass transfer during culture of highly metabolic and sensitive cell types, direct perfusion bioreactors for to have medium or cell solutions flow throughout a tissue or scaffold and bioreactors that provide controlled mechanical forces to the tissue [17].

Utilizing controlled mechanical forces has become the most popular for rapid fabrication techniques because mechanical methods offer the ability to deliver mechanical cues that tissues experience in the physiological setting. Mechanical forces used to induce maturation include pulsatile radial strain, longitudinal stretch, pulsatile flow, and compression [17-22]. These forces can be precisely controlled and generated using software, like LABVIEW, paired with stepper motors, pumps and actuators. Mechanistic variables include frequency, magnitude, time per day of stimulation, and total days of stimulation. Research has shown that these mechanical methods have successfully accelerated tissue maturation in terms of ECM deposition and increased mechanical properties for tissues based on cell sheet technologies and collagen gel tissue constructs $[18,19,21]$. Cyclic radial stretching and pulsatile flow have been shown to enhance ECM production (collagen and elastin) and enhance mechanical properties of tissue engineered blood vessels, but the parameters for optimal maturation (frequency, magnitude, time of stimulation) have not been defined [19-21,23,24]. Both static and dynamic longitudinal stretch of tissue sheets have accelerated extracellular matrix deposition and rapidly improve mechanical properties, both of which are critical for the development of a suitable tissue engineered replacements $[18,25]$.

Recently, magnetic forces have been investigated for mechanical conditioning with 'action at a distance.' By taking advantage of cells and ECM tagged with MNPs, RT-PCR results showed that dynamic magnetic forces ( $1 \mathrm{hr} /$ day, $1 \mathrm{wk}, 1 \mathrm{~Hz})$ led to upregulation of osteocalcin, osteopontin and alkaline phosphatase in human bone cells, when compared to controls [26]. Using magnetic forces for maturation offer several advantages: (1) precise control of physical stresses through variation of the magnetic force; (2) the MNPs are remotely coupled to a magnet array with no components connected inside the bioreactor, reducing potential contaminations; (3) magnetic arrays are scalable and present the opportunity to apply varying load profiles via particles and magnets of differing magnetic properties [26].

Despite advances in tissue maturation, standardized methods have not been developed for large scale applications and a challenge remains for understanding what mechanisms drive in vitro tissue maturation. 


\section{Current Challenges in Pre-processing, Processing and Post-Processing of Spheroids}

Poor mechanical properties, a lack of understanding fusion and maturation mechanisms, and the absence of engineered vascular networks creates uncertainty for fabricating viable and functional engineered tissues and organs. While long term viability of individual spheroids has been confirmed up to 7 weeks, the long term viability of larger tissues composed of spheroids is of concern due to the need for a method of nutrient and waste exchange with increasing tissue depth [15]. The ideal cell number and ECM content of spheroids for rapid tissue fabrication has not been elucidated and will likely be dependent on the application. A typical tissue engineered construct requires 107 to 109 cells, which means upscaling and automating spheroid production is required to be able to fabricate tissues and organs $[1,7]$.

Understanding and accelerating the fusion of spheroids is essential for the success of tissue fabrication. High throughput testing of maturogenic factors on fusing spheroids, as well as studying the influences of cell number and ECM content on fusing spheroids is providing quantitative data for understanding the mechanisms of this maturation process [27]. Work by Hajdu et. al. demonstrated that tissue spheroids incubated with maturogenic factors, TGF $\beta 1$ and serotonin, were more cohesive than control spheroids and on average were enveloped $40 \%$ by untreated spheroids, versus $10 \%$ by control spheroids [27]. Results also showed that these maturogenic factors had increased levels of collagen production as exhibited by increased immunofluorescent levels of Hsp47, a known intracellular chaperone of collagen types I-IV [27].

Magnetic forces have successfully been used to align magnetic cellular spheroids and accelerate their fusion by promoting active contacts between cells [13-15]. This technology has promise in developing technologies because physical forces generated by the magnetic field are able induce maturation without coming into contact with the tissue.

Currently, mechanical post-processing methods for maturing fused spheroids are in the early stages of development. Obtaining measurable mechanical properties of fusing and maturing tissues over time will lend insight for optimizing post-processing maturation techniques. Ideally, engineered tissues should contain the necessary cell types, ECM content and mechanical properties that would be required for functionality in vivo. Rapidly fabricated tissues should be available for use after maturation, or be stored long term for future needs. Preventing the unwanted fusion of spheroids stored for long term use must also be addressed.

The pre-processing, processing and post-processing steps in rapid tissue fabrication with spheroids have limitations that require improvements. Upscaled spheroid production, accelerating the fusion of spheroids with magnetic forces and improvements in bioreactor design give promise for using spheroids in rapid tissue fabrication. These emerging bioprocessing techniques must meet regulatory requirements that will emerge from their development to ensure their safety and efficacy in clinical applications. The lack of an engineered vascular network is the limiting factor in building tissues and organs with spheroids and must be addressed moving forward.

\section{References}

1. Mironov V, Visconti RP, Kasyanov V, Forgacs G, Drake CJ, et al. (2009) Organ printing: Tissue spheroids as building blocks. Biomaterials 30: 2164-2174.

2. Jakab K, Norotte C, Damon B, Marga F, Neagu A, et al. (2008) Tissue engineering by self-assembly of cells printed into topologically defined structures. Tissue Eng Part A 14:413-21.

3. Liver Transplants (2014) American Association for the study of Liver Diseases 20: 253-399.

4. Organ Donation and Transplantation Statistics (2014) National Kidney Foundation.

5. Achilli TM, Meyer J, Morgan JR (2012) Advances in the formation, use and understanding of multi-cellular spheroids. Expert Opin Biol Ther12: 1347-1360.

6. Fleming PA, Argraves WS, Gentile C, Neagu A, Forgacs G, et al. (2010) Fusion of uniluminal vascular spheroids: A model for assembly of blood vessels. Dev Dyn 239: 398-406.

7. Mironov V, Kasyanov V, Markwald RR (2011) Organ printing: from bioprinter to organ biofabrication line. Curr Opin Biotechnol 22: 667-673.

8. Tung YC, Hsiao AY, Allen SG, Torisawa YS, Ho M, et al. (2011) Highthroughput 3D spheroid culture and drug testing using a 384 hanging drop array. Analyst 136: 473-478.

9. Kunz-Schughart LA, Freyer JP, Hofstaedter F, Ebner R (2004) The Use of 3-D Cultures for High-Throughput Screening: The Multicellular Spheroid Model. J Biomol Screen 9: 273-285.

10. Ivascu A, Kubbies M (2006) Rapid Generation of Single-Tumor Spheroids for High-Throughput Cell Function and Toxicity Analysis. Journal of Biomolecular Screening 11: 922-932.

11. Mironov V, Prestwich G, Forgacs G (2007) Bioprinting living structures. J Mater Chem 17: 2054-2060.

12. Mironov V, Kasyanov V, Markwald RR (2011) Organ printing: from bioprinter to organ biofabrication line. Curr Opin Biotechnol 22: 667-673.

13. Mattix B, Olsen TR, Gu Y, Casco M, Herbst A, et al. (2014) Biological magnetic cellular spheroids as building blocks for tissue engineering. Acta Biomaterialia 10: 623-629.

14. Mattix B, Olsen TR, Moore T, Casco M, Simionescu D, et al. (2014) Accelerated Iron Oxide Nanoparticle Degradation Mediated by Polyester Encapsulation within Cellular Spheroids. Advanced Functional Materials 24: 800-807.

15. Mattix BM, Olsen TR, Casco M, Reese L, Poole JT, et al. (2014) Janus magnetic cellular spheroids for vascular tissue engineering. Biomaterials 35: 949-960.

16. Butler DL, Hunter SA, Chokalingam K, Cordray MJ, Shearn J, et al. (2009) Using functional tissue engineering and bioreactors to mechanically stimulate tissue-engineered constructs. Tissue Eng Part A 15: 741-749.

17. Martin I, Wendt D, Heberer M (2004) The role of bioreactors in tissue engineering. Trends in Biotechnology 22: 80-86.

18. Gauvin R, Parenteau-Bareil R, Larouche D, Marcoux H, Bisson F, et al (2011) Dynamic mechanical stimulations induce anisotropy and improve the tensile properties of engineered tissues produced without exogenous scaffolding. Acta Biomaterialia 7: 3294-3301.

19. Seliktar D, Black RA, Vito RP, Nerem RM (2000) Dynamic mechanical conditioning of collagen-gel blood vessel constructs induces remodeling in vitro. Annals of Biomedical Engineering 28: 351-362.

20. Seliktar D, Nerem RM, Galis ZS (2003) Mechanical strain-stimulated remodeling of tissue-engineered blood vessel constructs. Tissue Eng 9: 657-666.

21. Schutte SC, Chen Z, Brockbank KG, Nerem RM (2010) Cyclic strain improves strength and function of a collagen-based tissue-engineered vascular media. Tissue Eng Part A 16: 3149-3157. 
Citation: Olsen TR, Alexis F (2014) Bioprocessing of Tissues using Cellular Spheroids . J Bioproces Biotechniq 4: e112. doi:

Page 4 of 4

22. Seifu DG, Purnama A, Mequanint K, Mantovani D (2013) Smalldiameter vascular tissue engineering. Nat Rev Cardiol 10: 410-421.

23. Kim BS, Nikolovski J, Bonadio J, Mooney DJ (1999) Cyclic mechanical strain regulates the development of engineered smooth muscle tissue. Nat Biotech 17: 979-983.

24. Niklason LE, Gao J, Abbott WM, Hirschi KK, Houser S, et al. (1999) Functional Arteries Grown in Vitro. Science 284: 489-493.

25. Kolpakov V, Rekhter MD, Gordon D, Wang WH, Kulik TJ (1995) Effect of Mechanical Forces on Growth and Matrix Protein Synthesis in the In
Vitro Pulmonary Artery: Analysis of the Role of Individual Cell Types. Circulation Research 77: 823-831.

26. Dobson J, Cartmell SH, Keramane A, El Haj AJ (2006) Principles and Design of a Novel Magnetic Force Mechanical Conditioning Bioreactor for Tissue Engineering, Stem Cell Conditioning, and Dynamic in Vitro Screening. NanoBioscience, IEEE Transactions on 5: 173-177.

27. Hajdu Z, Mironov V, Mehesz AN, Norris RA, Markwald RR (2010) Tissue spheroid fusion-based in vitro screening assays for analysis of tissue maturation. J Tissue Eng Regen Med 4: 659-664. 